\title{
El diagnóstico del cáncer oral en el paciente geriátrico
}

\section{Diagnosis of oral cancer in the geriatric patient}

\author{
Jané Salas E*, Jané Pallí E**, Estrugo Devesa A*, Roselló Llabrés X*, \\ López-López J*
}

\section{RESUMEN}

El cáncer oral es una patología frecuente y creciente en el mundo occidental, su incidencia se incrementa año tras año, a pesar de que su localización, en una cavidad fácilmente accesible y explorable permitiría un diagnóstico temprano del mismo, o de las alteraciones potencialmente malignas. Las cifras de nuestro país sitúan una incidencia de 6,7 casos nuevos al año por 100.000 habitantes, con una proporción hombre/mujer de 3 a 1. La proporción de estas neoplasias aumenta de forma gradual con la edad, la posibilidad de que una lesión ulcerosa o proliferativa de cavidad oral, sea un carcinoma es mucho más elevada en pacientes mayores de 65 años. Es nuestra obligación el concienciar a nuestros pacientes, en el cese de hábitos nocivos y en la educación para establecer de la autoexploración una actividad habitual. Sin duda, una exhaustiva exploración física es la primera medida diagnóstica que poseemos asociada ante la mínima sospecha a la realización de una biopsia y el correspondiente estudio histopatológico, que puede establecer la potencialidad maligna de la lesión, así como la evolución de la misma. Disponemos, en la actualidad, de métodos complementarios de diagnósticos, que desde los tradicionales colorantes vitales, pasando por métodos ópticos, hasta la utilización de novedosas técnicas de identificación de receptores moleculares, de alteraciones cromosómicas podrían ser de utilidad en la orientación de factores de riesgo en pacientes predispuestos a padecer la patología, todo ello con el futuro puesto en identificar todos estos riesgos potenciales con un simple análisis de saliva. Lo cierto es que a día de hoy el mejor diagnóstico es la prevención y en el peor de los casos realizarlo lo más precozmente posible para mejorar las perspectivas vitales y la calidad de vida de nuestros pacientes.

Palabras clave: Cáncer, precáncer, diagnóstico precoz, paciente geriátrico.

\section{SUMMARY}

Oral cancer is a common and growing in the Western world disease, its incidence is increasing year after year, despite its location in an easily accessible and searchable cavity allow early diagnosis thereof, or of potentially malignant disorders. The figures of our country placed an incidence of 6.7 new cases per year per 100,000 population, with a male /female ratio of 3 to 1 . It is our duty to educate our patients in the cessation of harmful habits and the Education to establish how to examine a common activity. Definitely a thorough physical examination is the first diagnostic measure we have associated under suspicion for a biopsy and the corresponding histopathology, you can set the malignant potential of the lesion, and the evolution of it. We currently have complementary diagnostic methods, which based from traditional vital dyes, through optical methods, to the use of novel techniques for identifying molecular receptors, chromosomal alterations could be useful in guiding risk factors Patients predisposed to the disease, all in the future on identifying these potential

* Universidad de Barcelona. Facultad de Odontología. Departamento de Odontoestomatología. L'Hospitalet de Llobregat, Barcelona, España. Oral Health and Masticatory System Group (Bellvitge Biomedical Research Institute) IDIBELL, L'Hospitalet de Llobregat.

** Universidad de Barcelona. Facultad de Odontología. Departamento de Odontoestomatología. L'Hospitalet de Llobregat, Barcelona. 
risks with a simple analysis of saliva. The truth is that today the best diagnosis is prevention and the worst do it as early as possible to improve the life prospects and quality of life of our patients.

Key words: Cancer, precancer, early diagnosis, geriatric patient.

Fecha de recepción: 2 de mayo 2015.

Aceptado para publicación: 6 de mayo 2015.

Jané Salas E, Jané Pallí E, Estrugo Devesa A, Roselló Llabrés X, López-López J. El diagnóstico del cáncer oral en el paciente geriátrico. Av. Odontoestomatol 2015; 31 (3): 181-190.

\section{INTRODUCCIÓN}

El carcinoma oral de cabeza y cuello (COCE) es una neoplasia maligna de características particulares, que puede aparecer a cualquier edad, con incidencia que aumenta con la edad del paciente (Figs. 1 y 2), tiene clásicamente unos factores de riesgo conocidos y estudiados, el cese de estos hábitos nocivos podría tener un efecto positivo en la prevención de esta entidad. El tabaco, alcohol, la falta de higiene oral son circunstancias directamente relacionadas con su aparición. En fechas recientes, han aparecido otros factores de orden biológico, entre ellos ocupa un papel preponderante el virus del papiloma humano (VPH), que puede desempeñar un papel determinante en la aparición del COCE. Tiene particularidades epidemiológicas (afecta a pacientes más jóvenes), clínicas (afecta orofaringe) y pronósticos determinados (mejor pronóstico) (1).

Epidemiológicamente podemos buscar datos de incidencia en la página de Globocan (2) que permite cálculos pronóstico; pues bien, para España, y el año 2015, se pronostican 4.336 nuevos casos, siendo 2.564 en mayores de 65 años y, en esta franja de edad, se establece que la proporción hombre mujer es de 2 a 1, diferencia importante en el sector de población menor de 65 años en el que la proporción se establece en 3 o 4 a 1.

El paciente geriátrico tiene una serie de características diferenciadoras cuando sufren esta patología. En especial un retraso en el diagnóstico, ocasionado por la falta de hábito en la autoexploración y además suelen considerar que la presencia de úlceras orales puede deberse al uso de las prótesis. Por otro lado también influye negativamente la co-morbilidad que presentan (diabetes, patología cardiovascular, insuficiencia respiratoria, déficits de movilidad, etc.), los

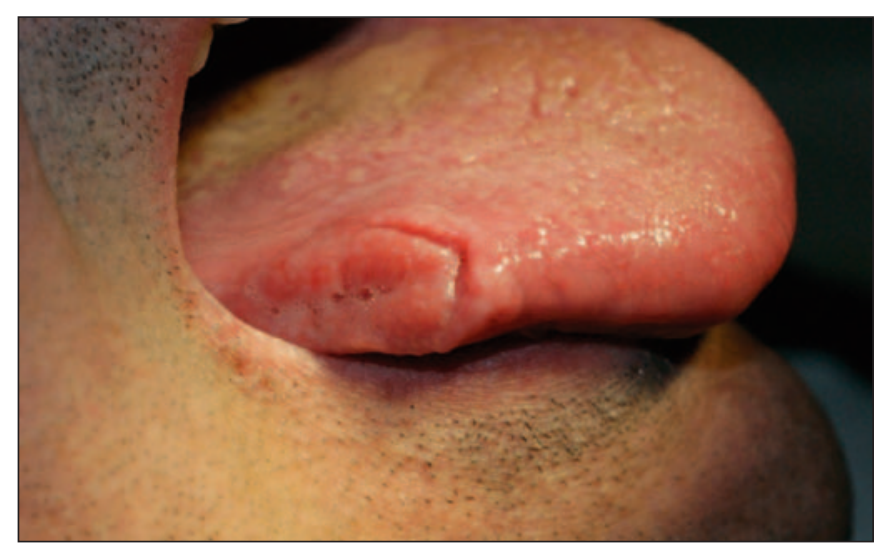

Fig. 1. Carcinoma lingual en borde lateral de lengua.

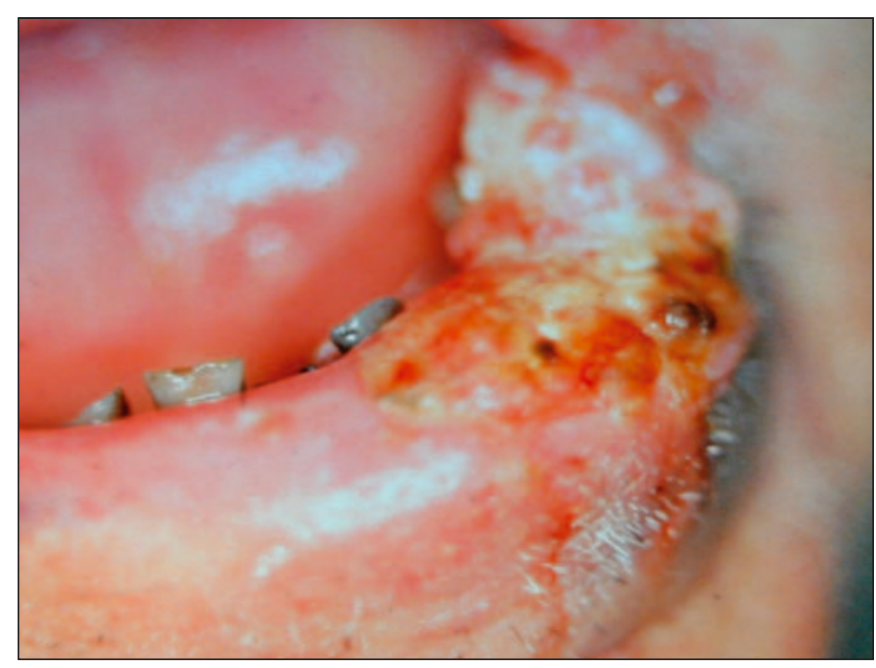

Fig. 2. Carcinoma labial de varios meses. 
déficits nutricionales, la menor respuesta a los tratamientos quimio-radioterápicos, los déficits cognitivos, el aumento importante de las complicaciones de las técnicas terapéuticas utilizadas y en algunos casos la ausencia del apoyo familiar que precisa todo enfermo oncológico (3-5). Nuestros pacientes mayores deben ser explorados, quizás con mayor insistencia en busca de procesos proliferativos en su cavidad oral, ya que la proporción de dicho cáncer va aumentando de forma aritmética con la edad (5). El diagnóstico diferencial se impone como imprescindible en el paciente de edad avanzada (6). A tal efecto el estudio de Mazyka y cols., refiere que en las biopsias realizadas en cavidad oral en pacientes con lesiones y mayores de 85 años, el $18,7 \%$, resultan malignas, frente al $11,7 \%$ en la franja de edad de 65 a 84 años (7).

La presentación de esta patología no es uniforme, y permite un curso evolutivo en la mayoría de los casos. Independientemente de los cambios a nivel ultraestructural que aparecen en las células en transición hacia un crecimiento que no es regulable y con pérdida progresiva de capacidad apoptótica, estos cambios se traducen en una serie de alteraciones histológicas que a su vez provocaran la aparición de lesiones evidenciables clínicamente. En el año 2007, Warnakurasuriya y cols. (8) describen una serie de lesiones que denominan alteraciones potencialmente malignas (Tabla 1), cambiando el criterio previo de las lesiones y condiciones premalignas estableci-

\section{TABLA 1.- ALTERACIONES POTENCIALMENTE MALIGNAS SEGÚN WARNAKULASURIYA 2007 (8)}

Alteraciones potencialmente malignas

- Leucoplasia.

- Eritroplasia.

- Lesiones palatinas en fumadores inversos.

- Fibrosis submucosa oral.

- Queratosis actínica.

- Liquen plano.

- Lupus eritematoso discoide.

- Disqueratosis congénita.

- Epidermolisis bullosa. do por la OMS, este cambio lo basan en los siguientes hechos:

A) Estudios longitudinales de pacientes que presentan ciertas alteraciones clínicas identificadas como "precancerosas" han evolucionad a cáncer en su seguimiento.

B) Algunas alteraciones, sobre todo las placas blancas y rojas coexisten en los márgenes de los carcinomas escamosos de la boca.

C) Una proporción importante de cambios morfológicos, citológicos, cromosómicos, genómicos y moleculares que se ven en el cáncer invasivo. También se detectan en estas lesiones precursoras.

Basándonos en estos conceptos, podemos afirmar que las lesiones invasivas no aparecen de "novo" (Fig. 3 ) en la cavidad oral, este es el motivo que debe hacer de la exploración por parte del profesional y de la autoexploración por parte del paciente la mejor herramienta para el diagnóstico de esta patología, en el caso de los pacientes ancianos esta exploración debe ser mucho más exhaustiva, ya que un diagnóstico precoz hace que el tratamiento sea lo menos agresivo posible. Este es el hecho fundamental que garantizará una mejora en la supervivencia de esta entidad. Recordemos que, a pesar de la capacidad técnica que poseemos, la supervivencia del cáncer de cavidad oral no supera el $50 \%$ a los 5 años y este porcentaje empeora en los pacientes de más edad (7).

Disponemos en la actualidad de una gran variedad de métodos complementarios que se ofertan para

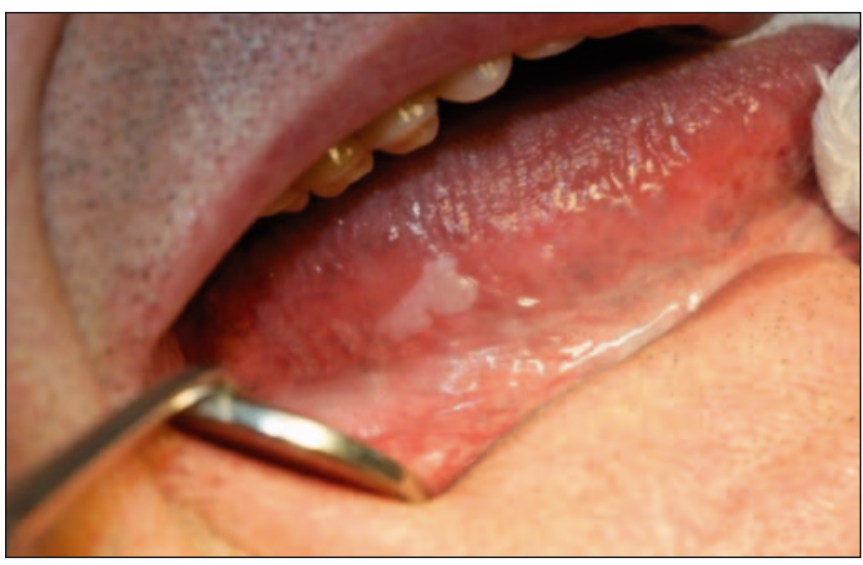

Fig. 3. Leucoplasia homogénea borde lingual de 6 meses evolución. 
mejorar la capacidad diagnóstica del cáncer de cavidad oral, todos ellos serán tratados a continuación (Tabla 2).

\section{BIOPSIA}

Es la obtención de una muestra de tejido tributario a ser diagnosticado desde el punto de vista histológico (Fig. 4), estableciendo las características de alte-

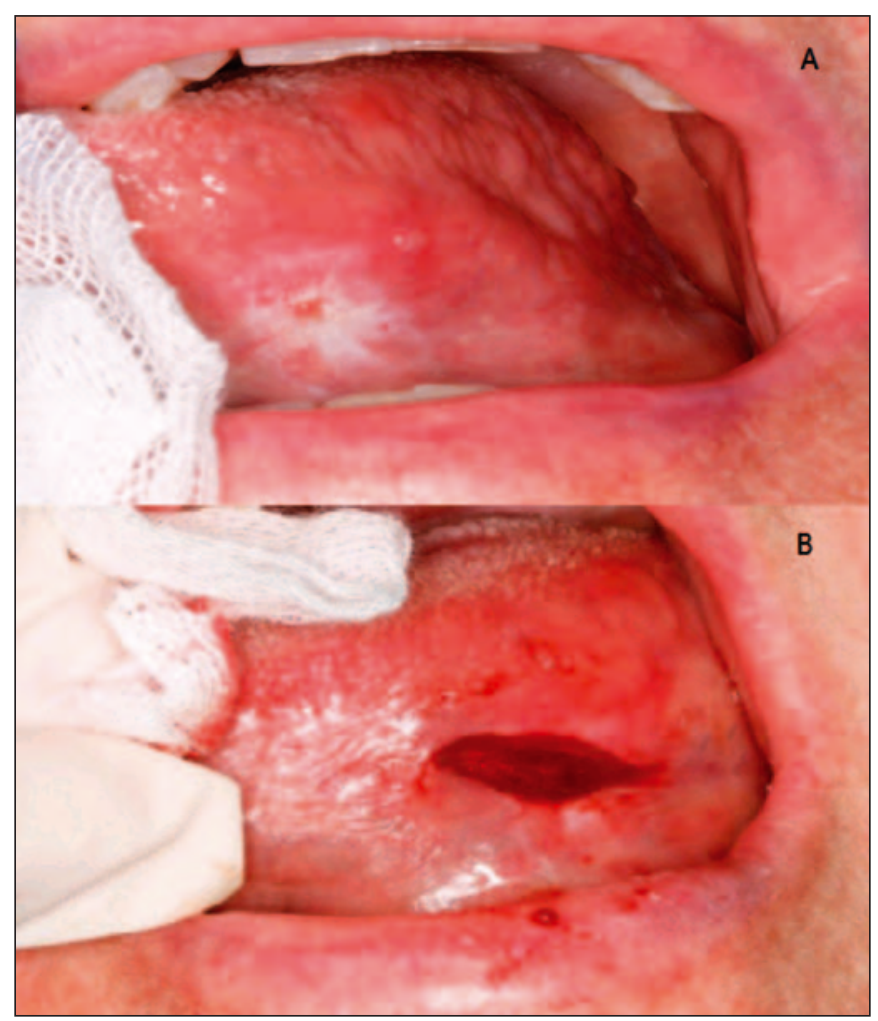

Fig. 4. A) Leucoplasia ulcerado en zona lateral de lengua de 15 días de evolución. B) Biopsia incisional.

\section{TABLA 2.- MÉTODOS DIAGNÓSTICOS COCE}

\begin{tabular}{|l|l|}
\hline Utilidad contrastada & Utilidad complementaria \\
\hline - Biopsia & - Citología exfoliativa. \\
& - Colorantes vitales. \\
& - Técnicas ópticas. \\
& - Alteraciones cromosómicas. \\
& - Marcadores moleculares. \\
& - Biomarcadores salivales. \\
\hline
\end{tabular}

ración, extensión y zona de transición con respecto al tejido que le rodea de características macroscópicas normales. Es el método diagnóstico que se utiliza como patrón de referencia para poder verificar la utilidad del resto de métodos existentes (9), el término que se utiliza es el de "gold standard". En la muestra se evalúa la disposición de maduración de las capas celulares dentro del epitelio, así como las características histológicas del tejido a examinar, permite un diagnóstico objetivo y tributario a ser considerado por otros profesionales, el problema surge en definir conceptos tales como la displasia y sus diferentes graduaciones, pero a pesar de ello es el método diagnóstico por excelencia ante casos sospechosos o para confirmar la evidencia clínica. No todos los profesionales están familiarizados con esta técnica y en ocasiones el material extirpado no siempre es derivado al estudio histopatológico correspondiente $(10,11)$, y en el caso de que se haga, en ocasiones el material obtenido no es suficiente o no es remitido en condiciones adecuadas. En las muestras obtenidas, además del estudio histológico clásico o de marcadores tumorales específicos, si la muestra no está fijada, pueden realizarse pruebas de inmunofluorescencia directa. La evidencia nos informa que existe un déficit en la formación de los graduados en odontología respecto a esta técnica. El problema reside no tan solo en la capacidad de realizar la técnica, ya que el paciente siempre podría ser derivado a un profesional más cualificado, sino en reconocer las lesiones que son tributarias a ser biopsiadas (12).

La citología exfoliativa podría ser considerada una técnica menor de biopsia. Se basa en las técnicas aplicadas en ginecología para prevención del cáncer en esta localización, la técnica comprende el raspado de la zona sospechosa, extensión y fijación en un portaobjetos que será remitido a un laboratorio centralizado, que mediante técnica informatizada generará un diagnóstico verificado por un citólogo. Esta técnica no debería utilizarse como diagnóstico de verificación ante la sospecha clínica de un carcinoma de cavidad oral, ni como método de despistaje ya que cada toma requiere la utilización de un nuevo procesamiento lo que podría encarecer de forma considerable su utilización. Cierto es que no todo es negativo, podría implementarse con técnicas inmunohistoquímicas que daría mayor sensibilidad a la 
técnica. Actualmente es un procedimiento que tiene utilidad en el seguimiento de zonas intervenidas de cáncer como control evolutivo y pronóstico del tratamiento realizado (13).

\section{COLORANTES VITALES}

Las apetencias cromáticas de la cromatina nuclear son conocidas desde hace tiempo. La fijación de ciertos colorantes ha sido utilizada para valorar zonas con procesos de crecimiento neoplásico, y a la vez para poder dirigir con mayor fiabilidad ante procesos extensos el lugar o los lugares donde realizar la biopsia. Dentro de los colorantes revisaremos los más utilizados: azules de metileno, toluidina, cloruro de tolonio, rosa de bengala y el lugol. Sólo comentaremos que en poblaciones altamente susceptibles a procesos neoplásicos de cavidad oral (India y sudeste asiático), se han puesto en marcha campañas de despistaje con enjuagues de ácido acético al $1 \%$, ya que en las zonas con procesos neoformativos aparecen más blanquecinas, este procedimiento permitiría derivar a centros de referencia a aquellos pacientes que presentan alteraciones con esta prueba.

\section{Azul de metileno, toluidina y cloruro de tolonio}

Existen estudios (14-16) que avalan esta técnica para localizar zonas sospechosas de las mucosas de la cavidad oral. Para que el resultado sea objetivable, el paciente debe primero enjuagarse con una solución de ácido láctico al $1 \%$ con agua destilada y saborizante (si se desea), en el caso de los ancianos es muy recomendable, durante $20-30$ segundos con el objetivo de eliminar residuos de alimentos y los excesos de saliva en la superficie a estudiar, posteriormente después de eliminar el enjuague el paciente realiza uno nuevo de azul de metileno al $1 \%$ durante 30 segundos, que será eliminado y volverá a enjuagarse de nuevo con el primer colutorio para eliminar los excedentes del colorante, haciendo evidente las zonas con más contenido cromático, destino de la biopsia a realizar. En los trabajos realizados, la sensibilidad oscila entre el 90-91,4\%, la especificidad entre el $66-69 \%$, con un valor predictivo positivo que oscila del 74 al $97 \%$ y un valor predictivo negativo del 33 al $87 \%$, estas cifras indican que es un método eficaz como parte de campañas de discriminación de lesiones de cavidad oral.

El cloruro de tolonio (17), también ha sido utilizado, presentando unos resultados de sensibilidad del $96,7 \%$ en algunos casos, a mayor grado de alteración tisular mayor porcentaje de positividad de la prueba.

\section{Rosa de Bengala}

Es un colorante ampliamente utilizado en el mundo de la oftalmología para el diagnóstico de las queratitis. Es el tetracloro [4,5,6,7] tetraiodo [2',4',5',7'] derivado de la fluoresceína. Se ha utilizado en un estudio piloto (18) para valorar su fijación en lesiones con aumento en la reproducción celular o con procesos inflamatorios, esto debe tenerse en cuenta en pacientes de edad avanzada, ya que la presencia de candidiasis podría enmascarar el resultado, la forma en cómo se realiza esta prueba tiene algunas diferencias con las practicadas en el caso del azul de metileno. El paciente se enjuaga con agua destilada un minuto, posteriormente se coloca con un algodón la solución de rosa de bengala sobre la zona a estudiar durante dos minutos, para finalizar el paciente volverá a enjuagarse con agua destilada por espacio de un minuto. Tras eliminar este último se procede a valorar la lesión mediante la comparación con una escala cromática, que va del rosa pálido hasta el granate, a mayor coloración, mayor probabilidad de lesión neoplásica que debería ser biopsiada. Este estudio refiere una sensibilidad de la prueba en el grupo estudiado (132 pacientes) del 93,9\% y una especificidad de 73,7\%. Los autores de este estudio concluyen que en los pacientes con leucoplasia o liquen plano con zonas displásicas o de carcinoma in situ, la prueba es muy sensible y permite orientar mucho mejor la zona a biopsiar.

\section{Lugol}

Este colorante vital es una solución iodada utilizada por primera vez por el médico francés Lugol (1829), consiste en la mezcla de $5 \mathrm{~g}$ de iodo, $10 \mathrm{~g}$ de ioduro potásico y $85 \mathrm{ml}$ de agua destilada. La técnica de utilización es semejante a la descrita para el rosa de 
bengala. En este caso, la prueba está destinada a la localización de lesiones en mucosa no queratinizada (19), no siendo tan efectiva en la queratinizada o en las mucosas más especializadas como el dorso lingual. Según Kujan y cols. (20), el yoduro de lugol es un método económico, fiable y rápido, en el análisis complementario de la cavidad oral en zonas sospechosas de degeneración tisular, no siendo peor que otros colorantes vitales ni los métodos de fluorescencia.

\section{TÉCNICAS ÓPTICAS}

La utilización de lentes para magnificar la visión de zonas a explorar y la utilización de filtros o fuentes de luz diferente a la blanca, han sido utilizados desde hace mucho tiempo en otros campos de la medicina, en concreto de la ginecología, que utiliza como método complementario a la exploración visual directa la colposcopia. Mediante ella el profesional visualiza las paredes vaginales y el cuello uterino, dependiendo de las características del epitelio observado en la zona de transición ecto-endocérvix, se establecen una serie de patrones colposcópicos que orientan el tipo de proceso subyacente que puede acontecer y que determinará la realización de la biopsia, guiada por algún colorante vital o no. Dentro de este proceder, Gynther y cols., en el año 2000 (21), publicaron un trabajo en el que proponían diferentes patrones vasculares subepiteliales que orientarían hacia cambios histopatológicos en la mucosa: No fue un trabajo que tuviese mucho predicamento hasta unos años más tarde en que surgen las técnicas de fluorescencia, en sus dos vertiente, auto y quimiofluorescencia. Uno de los inconvenientes común a las dos técnicas que comentamos es que precisan de una ausencia total de luz ambiental, que dependiendo de los lugares de trabajo no es fácil lograr.

\section{Autofluorescencia}

En el año 2006 aparece el primer artículo publicado en PubMed, refiriéndose a esta novedosa técnica complementaria de visualización de las mucosas $(22,23)$. El sistema Velscope ${ }^{\circledR}$ (Visually Enhanced Lesion Scope; LED Dental Inc., White Rock, B.C.). Es un dispositivo manual desarrollado por $L E D$ medical diagnostics en asociación con la Agencia de Cáncer British Columbia. Este sistema detecta la pérdida de fluorescencia en lesiones orales de alto riesgo, visibles o no al ojo desnudo, al aplicar fluorescencia directa. Consiste en una fuente de luz, que emite una longitud de onda de 400 a 460 nm y una unidad manual para la visualización directa. Bajo esta luz, la mucosa oral normal emite una autofluorescencia color verde, mientras que las áreas anormales absorben la luz fluorescente y se ven oscuras. Por lo tanto, detecta los cambios bioquímicos precozmente, antes de su apariencia evidente, lo que permite la detección temprana de lesiones patológicas. Emite una luz azul al interior de la cavidad oral, la cual excita los tejidos desde la superficie del epitelio, a través de la membrana basal y en el estroma adyacente, causando la fluorescencia. El concepto asociado a la autofluorescencia de los tejidos, es que cambios en la estructura y el metabolismo del epitelio, así como en el estroma subepitelial, alteran la interacción con la luz. Específicamente, estos cambios en el epitelio y en el estroma, pueden alterar la distribución en los tejidos de los fluoróforos, y como consecuencia la vía por la que emiten la fluorescencia después de la estimulación con una intensa excitación de luz azul. La hemoglobina absorbe fuertemente la luz autofluorescente producida por el colágeno y la elastina. Más específicamente el aumento de la presencia de sangre en la submucosa asociada a un aumento de la angiogénesis, puede resultar en absorción de colágeno y elastina producida por la luz autofluorescente, por lo que el área puede aparecer más oscura durante el examen. Hanken y cols. (24), en su artículo, concluyen que mejora la sensibilidad del diagnóstico, no así la especificidad y afirman que es una buena herramienta siempre que sea utilizada por un profesional experimentado (Fig. 5). En las personas de edad avanzada es una técnica que al ser poco invasiva podría ser un buen complemento para diferenciar lesiones proliferativas de aquellas de tipo inflamatorio.

\section{Quimiofluorescencia}

Vizilite, (Zila pharmaceuticals, Phoenix, Arizona, USA), es el sistema que se basa en la quimiofluorescencia, puede estar implementado con azul de toluidina (Vizilite-plus) (25). La técnica a utilizar es enjuague 


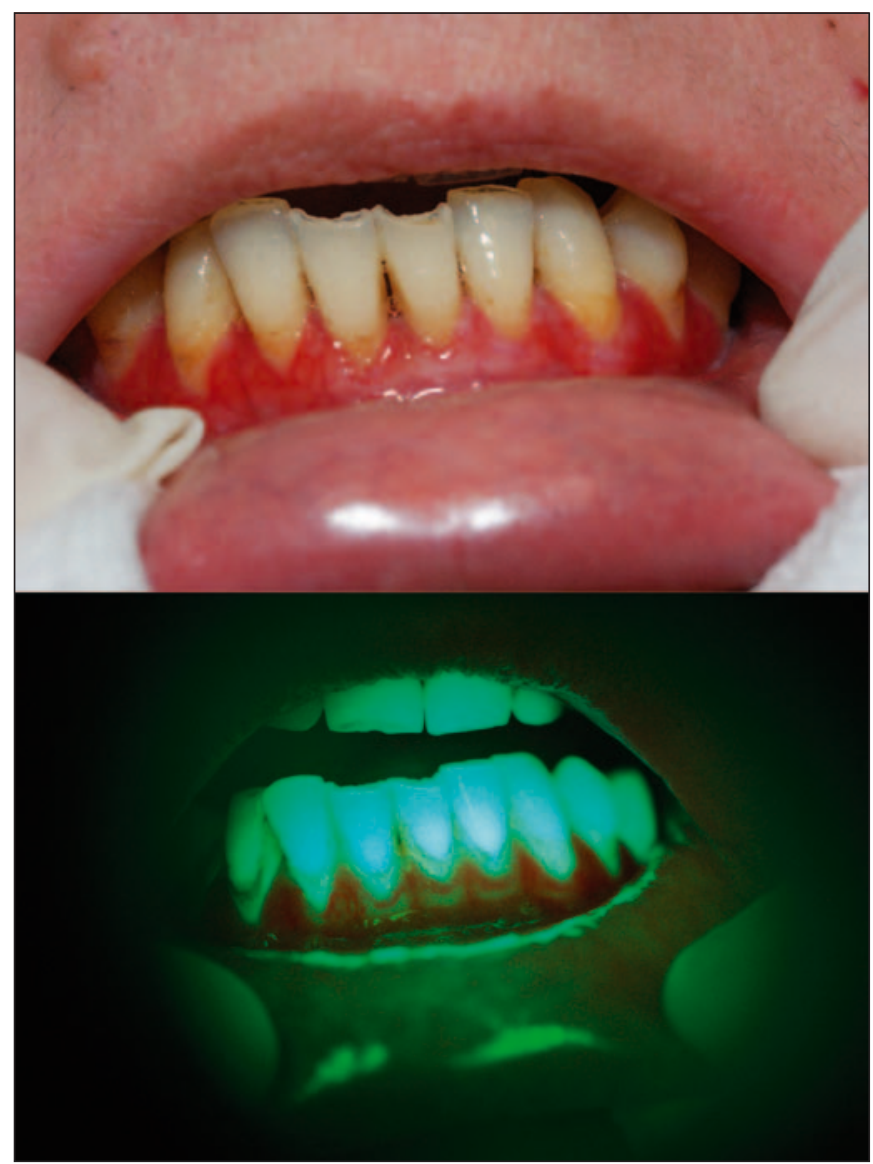

Fig. 5. Gingivitis descamativa e imagen obtenida mediante el Velscope ${ }^{\circledR}$.

previo a la visualización con ácido acético al $1 \%$ durante 1 minuto y, posteriormente, visualizar la cavidad oral con una lámpara de uso exclusivo para cada paciente, es de agitación, con lo que la duración de la exploración es limitada y un periodo óptimo que no supera los 3 minutos. Después, la lámpara se apaga y, para volver a explorar al paciente, se requiere otro kit. No todos los trabajos realizados con este sistema reflejan una efectividad que lo haga preferible a otros métodos complementarios. Así, Oh y cols. (26) concluyen que el ácido acético permite visualizar algunas lesiones adicionales respecto a la luz blanca convencional, pero que la lámpara específica genera reflejos en los tejidos y no es de utilidad para el explorador, comparando con la autofluorescencia posee menor sensibilidad, $87,1 \%$ versus $77,1 \%$.

Dentro de esto apartados podríamos considerar otro tipos de medios que se podrían utilizar, como el
Identafi ${ }^{\circledR}$, que permite en la misma lámpara utilizar tres longitudes de ondas diferentes. La dielectroforesis que permite calcular la resistencia de los tejidos, que se ven alteradas en el caso de las neoplasias y para finalizar la tomografía de coherencia óptica que permite observar las superficies epiteliales y trazar patrones de diferenciación patológica, al ser métodos poco contrastados y de poca aplicación práctica no van a ser desarrollados en esta revisión.

\section{ALTERACIONES CROMOSÓMICAS}

Hoy está bien establecido que la aparición de lesiones en la cavidad oral suele ser como consecuencia de cambios en las estructuras tisulares que proceden a su vez de cambios celulares (debidas a problemas o alteraciones que suceden a nivel de las estructuras que integran los núcleos, es decir los cromosomas) (27). El ADN que se aísla en las células neoplásicas puede presentar numerosas modificaciones: pérdida de heterocigosidad, inestabilidad cromosómica, mutaciones puntuales y cambios epigenéticos de diferentes oncogenes y genes supresores. Estos cambios podrían ser utilizados de forma fiable para detectar lesiones precancerosas. En el laboratorio se puede proceder a procesos de amplificación de segmentos de cromosoma y estos son portadores de oncogenes que se significan en locis concretos en el caso del cáncer oral: 11q13 (CCND1: ciclina D1, la proteína fosfatasa $1 \alpha$ ), 7p13-22 (EGFR), 8q24 (myc), y 18q21 (BCL-2). La pérdida de heterocigosidad hace perder alelos de genes supresores, esta falta de material genético puede predisponer a alteraciones que se dirigen hacia la falta de capacidad apoptótica por parte de las células afectadas. Estos cambios pueden observarse en la estructura cromosómica de las leucoplasias y de las displasias de diferentes grados. En el caso de los COCE, la pérdida de material genético se observa en CDKN2A (p16INK4A), p14 (ARF), p53, y APC. Las regiones cromosómicas 3p14 (FHIT), 3p25 (BVS), 4q, 8p21-23, 9p21-2260, 13q14 (PRB), 17p13.1 (p53), y 18q21.1 (DCC) son las principales localizaciones de heterocigosidad del COCE. La edad de los pacientes no parece ser un diferencial en las expresiones de estas alteraciones.

Los cambios epigenéticos como la metilación, son comunes en la tumorogénesis, ciertas regiones (is- 
las) de ADN son ricas en citosina-fosfato guanosina (CPG). Se conocen tres metiltransferasas del ADN, capaces de metilar las islas de CPG, estas zonas se encuentran en muchas regiones promotoras de algunos genes supresores tumorales. La metilación puede provocar la inactividad de la transcripción de estas moléculas. Genes que se afectan con frecuencia son CDKN2A (p16INK4A), p14ARF, CDH1, MGMT, CHFR, y la proteína quinasa asociada a la muerte celular (DAPK1). Del mismo modo, otro estudio mostró que la metilación aberrante de la EDNRB, KIF1A y DCC es común en pacientes con cáncer oral. Por otra parte, la hipermetilación promotora de reparación de genes (tales como genes hMLH1 y hMSH2) se ha encontrado en el cáncer oral.

\section{MARCADORES MOLECULARES}

Una de las características de las células tumorales, como hemos comentado, es que presentan alteraciones cromosómicas que a su vez provocan la alteración o aparición de cambios de estructuras moleculares. Estas alteraciones pueden ser determinadas en el laboratorio mediante técnicas inmunohistoquímicas que se practican en muestras obtenidas por biopsia, y en menor medida por citología exfoliativa. Bagán en su libro establece que los biomarcadores se pueden clasificar según se muestra en la Tabla 3 (28).

Existen estudios recientes con nuevos biomarcadores que pueden ser utilizados como factores pronós-

TABLA 3.- CLASIFICACIÓN DE LOS BIOMARCADORES SEGÚN BAGÁN JV (28)

- Actúan en la proliferación celular:
EGFR, Ciclina D1, Ki 67, PCNA y Akt1.

- Actúan en la supresión y apoptosis celular: p53/63, p21/27, pRB, Bcl-2.

- Biomarcadores que miden la hipoxia: HIF-1 $\alpha$, anhidrasa carbónica IX y EPOR.

- Los que actúan sobre la angiogénesis. VEGF, receptor de la tirosin kinasa (Eph)

- Marcadores de la adhesión celular: Cadherina, cateninas y CD44, entre otros. tico de progresión de displasias hacia un cáncer invasivo (29). Sin duda esto representa un gran avance en el pronóstico evolutivo del cáncer oral. Los autores analizan la sobreexpresión de la proteína S100A7 y concluyen que esta proteína sobreexpresada es un indicador de degeneración neoplásica de las displasias.

\section{BIOMARCADORES SALIVALES}

La saliva es un fluido orgánico de fácil recolección, evaluable con facilidad, que permite la detección de marcadores diferentes a los obtenidos por fragmentos de tejido, en el seguimiento de enfermedades, e incluso en el seguimiento de pacientes adictos a drogas y en programa de deshabituación. Es un enfoque prometedor y desde hace años hay investigadores que buscan elementos que permitan de forma precoz diagnosticar el inicio o la progresión de las alteraciones potencialmente malignas hacia el cáncer. Chen y cols. (30), revisan todos los biomarcadores salivales propuestos desde un punto de vista experimental hasta 2013. Sin duda la cantidad es ingente y difícil de poder evaluar. Muchas de las substancias buscadas utilizan diferentes medios de diagnóstico, quizás uno de los que se repite con más asiduidad es la valoración de los niveles de interleucina 8 (IL-8). Hay evidencias que indican que los niveles de esta interleucina está aumentada en saliva en pacientes con COCE, y no en lesiones premalignas, el problema es que en los pacientes con lesiones precancerosas y en sujetos control sin patología aparente no hay cambios significativos. Es una prueba que será de mucha utilidad en pacientes geriátricos, institucionalizados o no, dada su facilidad. Es un método diagnóstico de futuro, que probablemente permitirá avances diagnósticos y pronóstico en el cáncer de cavidad oral.

\section{CONCLUSIONES}

1. El COCE es una patología que aumenta en incidencia y proporcionalmente con la edad.

2. La prevención es la mejor opción, educando a nuestros pacientes en el cese de hábitos tóxicos y promulgando la autoexploración para que los pacientes sean conscientes de cambios que puedan aparecer en su boca. 
3. Es obligación del odontólogo generalista tener las habilidades básicas que permitan sospechar de la presencia de alteraciones potencialmente malignas y realizar la biopsia o derivarla en caso de que lo crea conveniente.

4. La irrupción de métodos complementarios de diagnóstico no debe dar sensación de falsa seguridad ante pruebas negativas, ya que estas tienen una elevada tasa de falsos positivos.

5. Los avances en genómica y en los estudios de marcadores salivales son prometedoras como herramientas de screening en un futuro próximo.

\section{BIBLIOGRAFÍA}

1. McLaughlin-Drubin ME, Meyers J, Munger K. Cancer associated human papillomaviruses. Current Opinion in Virology 2012;2:459-66.

2. Globocan. http://globocan.iarc.fr/. Accedido en 30 de marzo de 2105.

3. Brugel L, Laurent M, Caillet P, Radenne A, DurandZaleski I, Martin M, et al. Impact of comprehensive geriatric assessment on survival, function, and nutritional status in elderly patients with head and neck cancer: protocol for a multicentre randomised controlled trial (EGeSOR). BMC Cancer 2014; Jun 13;14:427.

4. Lange M, Rigal O, Clarisse B, Giffard B, Sevin E, Barillet $M$, et al. Cognitive dysfunctions in elderly cancer patients: a new challenge for oncologists. Cancer Treat Rev 2014;40:810-7.

5. Maggiore RJ, Curran EK, Witt ME, Haraf DJ, Vokes EE, Cohen EE. Survival and selected outcomes of older adults with locally advanced head/neck cancer treated with chemoradiation therapy. J Geriatr Oncol 2013;4:327-33.

6. Thomas JE. Differential diagnosis of oral lesions in geriatric patients. Todays FDA 2012;24:24-9.

7. Muzyka BC, Dehler KR, Brannon RB. Characterization of oral biopsies from a geriatric population. Gen Dent 2009;57:432-7.
8. Warnakulasuriya S, Johnson NW, van der Waal I. Nomenclature and classification of potentially malignant disorders of the oral mucosa. J Oral Pathol Med 2007;36:75-80.

9. Mota-Ramírez A, Silvestre FJ, Simó JM. Oral biopsy in dental practice. Med Oral Patol Oral Cir Bucal 2007;12:E504-10.

10. Kumaraswamy KL, Vidhya M, KumarRao P, Mukunda A. Oral biopsy: Oral pathologist's perspective. Journal of Cancer Research and Therapeutics 2012;8:192-8.

11. Seoane JM, González-Mosquera A, Velo-Noya J. La biopsia oral en el contexto del precáncer y del cáncer oral. Av. Odontoestomatol 2008;24:89-96.

12. M S, Shetty P, Decruz AM, Pai P. The Self-Reported Knowledge, Attitude and the Practices Regarding the Early Detection of Oral Cancer and Precancerous Lesions among the Practising Dentists of Dakshina Kannada-A Pilot Study. J Clin Diagn Res 2013;7(7):1491-4.

13. Bhoopathi V, Kabani S, Mascarenhas AK. Low positive predictive value of the oral brush biopsy in detecting dysplastic oral lesions. Cancer 2009; 115:1036-40.

14. Riaz A, Shreedhar B, Kamboj M, Natarajan S. Methylene blue as an early diagnostic marker for oral precancer and cancer. SpringerPlus 2013; 2:95.

15. Chen YW, Lin JS, Wu CH, Lui MT, Kao SY, Fong Y. Application of In Vivo Stain of Methylene Blue asa Diagnostic Aid in the Early Detection and Screening of Oral Squamous Cell Carcinoma and Precancer Lesions. J Chin Med Assoc 2007;70: 497-503.

16. Awan KH, Morgan PR, Warnakulasuriya S. Assessing the accuracy of autofluorescence, chemiluminescence and toluidine blue as diagnostic tools for oral potentially malignant disorders-a clinicopathological evaluation. Clin Oral Invest 2015; (Epub ahead of print). DOI 10.1007/s00784-015-1457-9. 
17. Epstein JB, Feldman R, Dolor RJ, Porter SR. The utility of tolonium chloride rinse in the diagnosis of recurrent or second primary cancers in patients with prior upper aerodigestive tract cancer. Head Neck 2003;25:911-21.

18. Du GF, Li CZ, Chen HZ, Chen XM, Xiao Q, Cao $Z G$, et al. Rose bengal staining in detection of oral precancerous and malignant lesions withcolorimetric evaluation: A pilot study. Int. J. Cancer 2007;120:1958-63.

19. Petruzzi M, Lucchese A, Baldoni E, Grassi FR, Serpico R. Use of Lugol's iodine in oral cancer diagnosis: An overview. Oral Oncology 2010; 46:811-3.

20. Kujan O, Glenny AM, Duxbury J, Thakker N, Sloan P. Evaluation of screening strategies for improving oral cancer mortality: a Cochrane systematic review. J Dent Educ 2005;69:255-65.

21. Gynther GW, Rozell B, Heimdhal A. Direct oral microscopy and its value in diagnosing mucosal lesions: a pilot study. Oral Surg Oral Md Oral Pathol Oral Radiol Endod 2000;90:164-70.

22. Kois JC, Truelove E. Detecting oral cancer: a new technique and case reports. Dent Today 2006; 25:96-7.

23. Trullenque-Eriksson A, Muñoz-Corcuera M, Campo-Trapero J, Cano-Sánchez J, Bascones-Martínez A. Analysis of new diagnostic methods in suspicious lesions of the oral mucosa. Med.Oral Patol.Oral Cir.Bucal 2009;14:E210-6.

24. Hanken H, Kraatz J, Smeets R, Heiland M, Assaf AT, Blessmann $M$, et al. The detection of oral pre- malignant lesions with an autofluorescence based imaging system (VELscope ${ }^{T M}$ ) - a single blinded clinical evaluation. Head Face Med 2013; 9:23.

25. Mojsa I, Kaczmarzyk T, Zaleska M, Stypulkowska J, Zapala-Pospiech A, Sadecki D. Value of the ViziLite Plus System as a diagnostic aid in the early detection of oral cancer/premalignant epithelial lesions. J Craniofac Surg 2012; 23:162-4.

26. Oh ES, Laskin DM. Efficacy of the ViziLite system in the identification of oral lesions J Oral Maxillofac Surg 2007;65:424-6.

27. Mishra R. Biomarkers of oral premalignant epithelial lesions for clinical application. Oral Oncology 2012;48:578-84.

28. Bagán JV. Medicina y Patología Bucal. 2013. Tema 20 Bagán JV, Díaz JM. 333-44. Valencia ISBN 978-84-616-5253-2.

29. Kaur J, Matta A, Kak I, Srivastava G, Assi J, Leong I, et al. S100A7 overexpression is a predictive marker for high riskof malignant transformation in oral displasia. Int. J. Cancer 2014;134:1379-88.

30. Chen YS, Rees T, Wright J. A review of research on salivary biomarkers for oral cancer detection. Clin Transl Med 2014;3:3.

\section{CORRESPONDENCIA}

\section{Enric Jané Salas}

Departamento de Odontoestomatología

Facultad de Odontología.

Universidad de Barcelona (Campus Universitario de Bellvitge)

08907 L'Hospitalet de Llobregat, Barcelona, España.

Correo electrónico: enricjanesalas@ub.edu 